\title{
Diagnostic approach and treatment specificity in patients with focal lesions of the biliopancreatic area complicated by mechanical jaundice
}

\author{
PETRO MURAVIOV ${ }^{1,2^{*}}$, BORIS ZAPOROZHCHENKO ${ }^{1,2}$, IGOR BORODAEV $^{1,2}$, V.V. KOLODIY ${ }^{1,2}$, \\ ANATOLIY GORBUNOV ${ }^{1,2}$, E.P. KIRPICHNIKOVA ${ }^{3}$, NICOLAE BACALBASA ${ }^{4,5^{*}}$, \\ IRINA BALESCU $^{6}$ and MAKREM HARHOURI ${ }^{1}$
}

\author{
${ }^{1}$ Department of Surgery, Odessa National Medical University, 65082 Odessa; \\ ${ }^{2}$ Department of Surgery, MUI 'Odessa Regional Clinical Medical Center' of the Odessa Regional Council, 79761 Odessa; \\ ${ }^{3}$ Department of Anesthesiology, Intensive Care and Emergency Medicine, Odessa National Medical University, \\ 65082 Odessa, Ukraine; ${ }^{4}$ Department of Obstetrics and Gynecology, 'Carol Davila' University of Medicine and Pharmacy, \\ 020021 Bucharest; ${ }^{5}$ Department of Visceral Surgery, Center of Excellence in Translational Medicine, \\ 'Fundeni' Clinical Institute, 022328 Bucharest; ${ }^{6}$ Department of Visceral Surgery, \\ 'Ponderas' Academic Hospital, 021188 Bucharest, Romania
}

Received August 9, 2021; Accepted September 8, 2021

DOI: $10.3892 / \mathrm{etm} .2021 .11082$

\begin{abstract}
Pancreatoduodenal resection (PDR) represents one of the most demanding procedures which is required in patients with a tumoral lesion at this level. The aim of the present article was to report the results of 272 patients submitted to such surgical procedures. All patients were divided into two groups: the main group, for whom the optimized decompression algorithm was used $(n=112)$ and the control group, for whom preparation for PDR was carried out according to generally accepted standards $(n=160)$. Upon admission to the hospital, the total bilirubin level in the main group was $274.6 \pm 5.9 \mu \mathrm{mol} / 1$ while in the control group this level was $270.4 \pm 4.6 \mu \mathrm{mol} / 1$. PDR was performed in 272 of the patients. Whipple's terminolateral anastomosis was performed in 38/160 (23.7\%) of patients in the control group; termino-terminal anastomosis according to Shalimov-Kopchak's method was imposed in 40/160 (25.0\%) patients of the control group. Pancreatojejunoanastomosis with
\end{abstract}

Correspondence to: Dr Petro Muraviov, Department of Surgery, Odessa National Medical University, 2 Valikhovsky Lane, 65082 Odessa, Ukraine

E-mail: gemostatik@gmail.com

*Contributed equally

Abbreviations: CEA, carcinoembryonic antigen; CA, cancer antigen; PDR, pancreatoduodenal resection; MRI, magnetic resonance imaging

Key words: jaundice, pancreatoduodenal resection, mortality, pancreatojejunoanastomosis, pancreatogastroanastomosis, biliary decompression the imposition of a ductomucosal anastomosis was performed in 128 patients in total with 73 cases in the main group and in 55 cases in the control one. Pancreatogastroanastomosis with plunging of the pancreatic stump into the stomach stump was performed in 35 patients in total with 19 patients in the main group and 16 in the control group. Pancreatogastroanastomosis with plunging of the pancreatic stump into the 'stomach sleeve' was performed in 32 patients in total with 20 patients in the main group and 12 in the control group. The mortality rate in the main group was $6.3 \%$ ( 7 patients), and in the control group, $11.9 \%$ (19 patients). In conclusion, biliary decompression may improve the postoperative outcomes after pancreatic resection.

\section{Introduction}

The main surgical procedure in the treatment of focal lesions of the pancreatoduodenal area, accompanied by obstructive jaundice, is pancreatoduodenal resection (PDR). It is one of the most complex surgical interventions and is accompanied by a large number of postoperative complications (1-3). Performing PDR in cases presenting obstructive jaundice increases the risk of postoperative complications up to 40-52\% and is accompanied by high postoperative mortality rates of $15-19 \%(1-5)$.

Meanwhile, the fact that the existing methods of biliary decompression are not safe enough and can lead to deterioration of the patient's condition during the first 4-5 days after procedure should be emphasized. This deterioration may be manifested by a progression of cholestasis and cytolysis syndrome, liver insufficiency, encephalopathy and even multiple organ failure $(3,4)$. Diagnosis of the cause of the obstructive jaundice, the possibility of predicting the course of the perioperative period, individual determination of resectability, as well as improving the patient quality of life after 
radical surgical treatment continue to be widely discussed and emphasize the urgency of the issue.

The aim of the present article was to optimize the approach of the diagnosis and surgical treatment of patients with focal lesions of the pancreatobiliary area complicated by obstructive jaundice.

\section{Patients and methods}

The analysis of complex surgical treatment of 272 patients with lesions of the pancreatoduodenal area accompanied by jaundice was carried out. These surgeries were carried out in the surgical department of the Odessa Regional Clinical Medical Center (Odessa, Ukraine) from 2007 to 2018. The average age of patients was $49.1 \pm 3$.6 years; there were 141 men (51.8\%), and 131 women $(48.2 \%)$.

In addition to the generally accepted diagnostic algorithm, it was mandatory to determine the levels of specific tumor markers [carcinoembryonic antigen (CEA), carbohydrate or cancer antigen (CA)19-9, CA50, CA242]. Pancreatic head cancer was identified in 174 patients (63.9\%), Vater's papilla cancer in 20 cases (7.4\%), distal bile duct cancer in 24 cases (8.8\%) and chronic pseudotumoral pancreatitis in 54 patients (19.9\%).

The resectability of the pathological process was analyzed by magnetic resonance imaging (MRI) cholangiography; the sensitivity and specificity of arterial invasion detection by this mean being 83.3 and $71.4 \%$, respectively; meanwhile the sensitivity and specificity of venous invasion detection was 50 and $42.9 \%$, respectively. In the majority of patients in this study, the process was resectable; therefore resection was performed in $72(64.3 \%)$ cases in the main group, in 99 (61.9\%) cases in the control group [8 (7.1\%) and 32 (20.0\%) patients with chronic pancreatitis in the main and control group, respectively]. Borderline resectable process was determined in $26(23.2 \%)$ of patients in the main group and $16(10.0 \%)$ of patients in the control group [3 (2.7) and 11 (6.8\%) patients, with chronic pancreatitis in the main and control group, respectively]. Contingently resectable process was verified in patients of stage IV; 3 (2.7\%) patients in the main group and 2 (1.3\%) patients in the control group (Table I).

In regards to the clinical symptoms, the leading roles were played by manifestations of obstructive jaundice, Courvoisier's syndrome and minor oncologic signs on the background of persistent pain syndrome. Mainly patients were of IIB stage of the oncological process.

Statistical analysis. Statistical analysis was performed by methods of nonparametric statistics using the package Statistica 10.0 applications (Dell StatSoft Inc.; license no. JPZ706E091603APACA-1). Data are expressed as M \pm SEM, where $M$ is the sample mean, and SEM is the standard error of the mean. The statistical significance of the difference between the independent groups was checked using the Mann-Whitney test. Correlation analysis was performed based on Spearman's correlation rank criterion. We computed $\mathrm{P}$-values and considered a difference as statistically significant at $\mathrm{P}<0.05$.

\section{Results}

All 22 patients were divided into two groups: the main group, where the optimized decompression algorithm was used $(\mathrm{n}=112)$ and the control group, where preparation for PDR was carried out according to generally accepted standards $(n=160)$. Most of the patients were admitted in a severe condition, with long-lasting jaundice (2-3 weeks or more) and with high bilirubin levels ( $>200 \mu \mathrm{mol} / \mathrm{l})$. Upon admission to the hospital, the level of total bilirubin in patients of the main group was $274.6 \pm 5.9 \mu \mathrm{mol} / 1$ (direct, $211.2 \pm 2.2 \mu \mathrm{mol} / 1$; indirect, $63.4 \pm 2.6 \mu \mathrm{mol} / 1$. In the control group, the level of total bilirubin was $270.4 \pm 4.6 \mu \mathrm{mol} / 1$ (direct, $206.3 \pm 2.4 \mu \mathrm{mol} / 1$; indirect, $64.1 \pm 2.6 \mathrm{mmol} / \mathrm{l})$.

After determination of the risk of the planned radical surgery according to our own formula (taking into account the various parameters of the pathological process), a decision was made regarding the method of the surgical treatment.

In patients of the main group, preparation for the radical surgical intervention consisted in express-detoxification according to the developed scheme. The method of express-detoxification included traditional transdermal decompression of the biliaric tree with $6 \mathrm{~F}$ or $9 \mathrm{~F}$ catheters under ultrasound guidance, followed by taking a specially selected pharmaceutical complex and plasmapheresis. The efficiency of decompression was assessed by the volumetric flow rate of bile flow through the drainage; the optimal target level was taken as $60-100 \mathrm{ml} / \mathrm{day}$, the most acceptable being $300 \mathrm{ml} / \mathrm{day}$. In order to avoid the phenomena of hepatargy, the decrease in the rate of bile secretion was regulated; the volumetric rate did not exceed $50 \%$ of the previous level, determined for $24 \mathrm{~h}$ before the current day. Additionally, markers of cytolytic and cholestatic syndromes were determined. A satisfactory decrement for these indicators was no more than $20-25 \%$ per day.

Due to the application of the developed detoxification algorithm with the use of biliaric decompression in patients of the main group on the third day the level of bilirubinemia averaged $185.1 \pm 2.4 \mu \mathrm{mol} / 1$, while on the fifth day it was already $163.2 \pm 2.6 \mu \mathrm{mol} / \mathrm{l}$ and on the day before PDR the level was $112.3 \pm 2.7 \mu \mathrm{mol} / 1$. In the control group, which followed the usual volume of patient preparation for radical surgery, the level of total bilirubin on the third day was $258.4 \pm 2.9 \mu \mathrm{mol} / 1$, on the fifth day it was $222.2 \pm 3.8 \mu \mathrm{mol} / 1$, and on the day before PDR the level was $198.3 \pm 3.3 \mu \mathrm{mol} / 1$. Thus, in the main group after decompression, the total bilirubin level decrement was $\Delta=-59.1 \%$, direct one was $\Delta=-62.5 \%$, indirect one was $\Delta=-47.6 \%$. While in the control group of patients on the day before PDR, the total bilirubin level decrement was $\Delta=-26.7 \%$, direct one was $\Delta=-27.2 \%$, indirect one was $\Delta=-25.0 \%$.

The terms before the beginning of the cholemia level decrease in patients of the main group was less ( 33 vs. $56 \mathrm{~h}$ ) than in patients in the control group (by 41.1\%), while the duration of the hyperbilirubinemia (or, more often, the achievement of its permissible limits for performing radical surgery limits) was significantly less (by 68.2\%) $(\mathrm{P}<0.01)$.

After preoperative preparation, $\mathrm{PDR}$ was performed in 272 of patients.

Whipple's terminolateral anastomosis was applied in $38 / 160$ (23.7\%) patients in the control group. Termino-terminal anastomosis according to Shalimov-Kopchak's method was imposed in 40/272 (14.6\%) of the patients with 40/160 $(25.0 \%)$ in the control group. Pancreatojejunoanastomosis with the imposition of a ductomucosal anastomosis was performed in 128/272 (47.0\%) of the patients with $73 / 112$ 
Table I. Patient distribution depending on the resectability.

\begin{tabular}{lccccc}
\hline \multirow{2}{*}{ Disease characteristic } & \multicolumn{2}{c}{ Main group $(\mathrm{n}=112)$} & & \multicolumn{2}{c}{ Control group $(\mathrm{n}=160)$} \\
\cline { 2 - 3 } & Malignant & Benign & & Malignant & Benign \\
\hline Resectable process & $72(64.3 \%)$ & $8(7.1 \%)$ & & $99(61.9 \%)$ & $32(20.0 \%)$ \\
Borderline resectable process & $26(23.2 \%)$ & $3(2.7 \%)$ & & $16(10.0 \%)$ & $11(6.8 \%)$ \\
Contingently resectable process & $3(2.7 \%)$ & - & & $2(1.3 \%)$ & - \\
Total & $101(90.2 \%)$ & $11(9.8 \%)$ & & $117(73.1 \%)$ & $43(26.9 \%)$ \\
\hline
\end{tabular}

Table II. Pancreatoduodenal resection reconstruction step variants.

Type of pancreatodigestive anastomosis

Whipple's terminolateral anastomosis

Shalimov-Kopchak's termino-terminal anastomosis

Pancreatojejunoanastomosis with the imposition of a ductomucosal anastomosis

Pancreatogastroanastomosis with plunging of the pancreatic stump into the stomach stump (terminolateral)

Pancreatogastroanastomosis with plunging of the pancreatic stump into the 'stomach sleeve'

Total

$\begin{array}{cc}\text { Main group n (\%) } & \text { Control group n (\%) } \\ - & 38(23.7 \%) \\ - & 40(25.0 \%) \\ 73(65.2 \%) & 55(34.4 \%) \\ 19(16.9 \%) & 16(10.0 \%) \\ 20(17.9 \%) & 12(7.5 \%) \\ 112 & 160 \\ 272(100 \%) & \end{array}$

(65.2\%) in the main group and 55/160 (34.4\%) in the control group. Pancreatogastroanastomosis with plunging of the pancreatic stump into the stomach stump was performed in $35 / 272(12.9 \%)$ patients with $19 / 112$ (16.9\%) of patients in the main group and 16/160 (10.0\%) in the control group. Pancreatogastroanastomosis with plunging of the pancreatic stump into the 'stomach sleeve' was performed in 32/272 (11.8\%) of the patients with 20/112 (17.9\%) of patients in the main group and 12/160 (7.5\%) patients in the control group (Table II).

In some of the patients from both groups, despite strict adherence to tactical and technical approaches, a number of postoperative complications were developed. It was the indication for performing second surgical interventions.

Minimally invasive puncture-drainage interventions were performed in 13/112 (11.6\%) of patients from the main group and in $21 / 160(13.1 \%)$ of the patients from the control group. The indication for their performance was the formation of various liquid accumulations.

In patients with profuse arrosive bleeding, surgical interventions pursued the aim of final (by stitching and ligation) or, more rarely, temporary (by tamponade) hemostasis in $4 / 112$ (3.6\%) of patients from the main group and 8/160 (5.0\%) of patients from the control group.

Relaparotomies were also performed in the case of progressive early adhesive intestinal obstruction development in $3(2.7)$ and $1(0.6 \%)$ of the patients from the main and control group, respectively.

In the cases of pancreato- or biliodigestive anastomosis failure and purulent complication development, mainly drainage laparotomic surgical interventions were performed in $5(4.5 \%)$ of the patients in the main group and in $13(8.1 \%)$ of patients in the control group.

Only in 3 cases was it necessary to perform relaparotomy with subsequent complete removal of the pancreatic stump and partial resection of the fistula carrying jejunum. Such interventions were induced mainly by complete failure of the pancreatodigestive anastomosis (PDA) and/or total necrosis of the pancreatic stump, when it was impossible to perform reconstructive surgery.

According to the Clavien-Dindo classification, postoperative complications were divided as follows: II grade (suppuration of the postoperative wound, postoperative gastrostasis, diarrhea, diabetes mellitus, cholangitis, failure of the PDA [type A and B according to the International Study Group for Pancreatic Fistula (ISGPF)] in 49 (43.8\%) patients from the main group and 74 (46.3\%) of patients from the control group; IIIa grade [failure of PDA (types A and B according to ISGPF), formation of bilomas and intraabdominal abscesses] in $13(11.6 \%)$ of patients from the main group and $21(13.1 \%)$ patients from the control group; IIIb grade [failure of pancreatodigestive (type $\mathrm{C}$ according to ISGPF) and biliodigestive anastomosis, arrosive bleeding, intraabdominal abscesses and adhesive intestinal obstruction] in 12 (10.7\%) of patients from the main group and 22 (13.8\%) of patients from the control group; grade IV (pancreatic stump necrosis with PDA failure and arrosive bleeding, biliodigestive anastomosis failure) in 11 (9.8\%) of patients from the main group and 10 (6.3\%) of patients from the control group.

The most common cause of the fatal complication development (Clavien-Dindo's, grade V) was pancreatic remnant (stump) necrosis with PDA failure, arrosive bleeding, sepsis 
Table III. Causes for fatal complications.

\begin{tabular}{lcc}
\hline Cause & Main group & Control group \\
\hline Pancreatic stump necrosis with partial PDA failure, sepsis and multiorganic failure & 3 & 5 \\
Pancreatic stump necrosis with partial PDA failure and arrosive bleeding & 2 & 3 \\
Biliodigestive anastomosis failure & - & 1 \\
Thrombosis of the superior mesenteric artery (grade V) & - & 1 \\
Thrombosis of the own hepatic artery (grade V) & 1 & 1 \\
Portal vein thrombosis (grade V) & - & 1 \\
Hepatargy (grade V) & 1 & 4 \\
Acute myocardial infarction (grade V) & - & 1 \\
Massive pulmonary embolism (grade V) & - & 2 \\
Total & $7(6.3 \%)$ & $19(11.9 \%)$ \\
\hline
\end{tabular}

PDA, pancreatodigestive anastomosis. Clavien-Dindo's, grade V.

and multiorganic failure in 13 cases: 5 (4.5\%) of patients from the main group and $8(5.0 \%)$ of patients from the control group (Table III).

More rare cases, which were not associated with the pancreatic remnant (stump) condition, were represented by acute myocardial infarction (1 patient in the control group), massive pulmonary embolism (2 patients in the control group), thrombosis of the own hepatic artery (1 patient in the main group and 1 patient in the control group), thrombosis of the superior mesenteric artery (1 patient in the control group), portal vein thrombosis (1 patient in the control group) and failure of biliodigestive anastomosis with the development of bile peritonitis (1 patient in the control group).

The death of 5 patients [1 $(0.9 \%)$ patient in the main group and $4(2.5 \%)$ patients in the control group] were caused by liver failure as a result of long-term jaundice.

Mortality rate in the main group was $6.3 \%$ (7 patients), and in the control group $11.9 \%$ (19 patients).

\section{Discussion}

Extended pancreatic resections have become the option of choice in the last few decades in order to achieve a good local control of the disease for biliopancreatic and duodenal malignancies $(6,7)$. For the present, improvement in the surgical techniques and association of complex gestures of vascular resections have led to the increase in proportions of resectability $(8,9)$. In addition, improved rates of long-term survival have been reported demonstrating in this way the efficacy and feasibility of the procedure. Meanwhile, it should be emphasized that pancreatic surgery is still associated with significant rates of postoperative complications as well as increased rates of perioperative death, one of the most important factors which seem to influence these parameters being represented by the biological status of the patient $(1,2)$.

Therefore, it has been widely demonstrated that association of jaundice represents a significant risk factor for developing further complications. Increased serum levels of bilirubin seem to induce coagulation disorders, immunological dysfunctions and impaired postoperative healing leading in this way to a higher risk of postoperative life threatening complications $(10,11)$.

In this respect, preoperative biliary drainage procedures in order to decrease bilirubin serum levels prior to resection have been widely investigated $(3,12-14)$. Initially it was considered that performing preoperative biliary drainage may increase the perioperative complication rates; however in the first meta-analysis which came to demonstrate this aspect, both patients submitted to resection and palliation were included (15). Another study conducted on the issue of the efficacy and safety of preoperative biliary drainage which was published in 2005 also suggest that performing such a procedure might increase the risks of postoperative complications; however, the authors underlined the fact that patients submitted to biliary drainage presented a poorer biological condition and a more advanced stage of the disease. Therefore, the higher rates of postoperative complications were rather related to these findings and not to the drainage itself (16).

Moreover, it seems that association of preoperative biliary drainage followed by jaundice resolution also represents a favorable prognostic factor regarding the early evolution of these patients. Therefore, in the study conducted by Smith et al and published in 2008, the authors introduced 155 patients submitted to pancreatoduodenectomy for pancreatic ductal adenocarcinoma and jaundice; among these cases 130 patients were submitted to preoperative biliary drainage followed by surgery while the other 25 were submitted to per primam resection. The authors demonstrated that lower serum levels of albumin, higher levels of alkaline phosphatase and higher levels of $\mathrm{C}$ reactive protein were associated with poorer survival rates while the preoperative serum levels of bilirubin did not impact the long term outcomes. However, the preoperative elevated serum levels of bilirubin seemed to negatively impact the early outcomes of these patients; therefore, all patients who were alive at six months preoperatively had reported a significant decrease in the bilirubin serum levels once the drainage had been performed; meanwhile the cases in which the preoperative drainage failed to prove its efficacy and who presented persistent increased levels of bilirubin reported a lower than six months survival (14). 
In conclusion, the use of a modified approach to the management of jaundiced patients with focal lesions of the pancreatobiliaric zone made it possible to prepare patients for PDR within a shorter period by achieving satisfactory indicators of their condition, to avoid progression of liver failure and to decrease twice the postoperative mortality rates.

\section{Acknowledgements}

Not applicable.

\section{Funding}

This research received no external funding.

\section{Availability of data and materials}

Further information regarding the data included in this paper are available upon request.

\section{Authors' contributions}

NB, PM, and BZ contributed to the conception of the study, collected, analyzed and interpreted data from the literature and critically revised the manuscript. VVK and AG contributed to the conception of the study, performed the literature research, drafted the manuscript and were responsible for confirming the authenticity of all the raw data. NB and IgB contributed to the conception of the study, performed the literature research, drafted the manuscript and were responsible for confirming the authenticity of all the raw data. IrB and MH contributed to the interpretation of the data from the literature, collected, analyzed and interpreted the data corresponding to the patients and critically revised the manuscript. SVS collected, analyzed and interpreted the data corresponding to the patients introduced in this study and critically revised the manuscript. All authors read and approved the final manuscript for publication.

\section{Ethics approval and consent to participate}

The Ethics Committee of Odessa National Medical University (Odessa, Romania) approved the study. Informed consent was obtained from all patients prior to surgery and all data were collected according to the principles of The Declaration of Helsinki.

\section{Patient consent for publication}

Not applicable.

\section{Competing interests}

There are no competing interests to declare regarding this study.

\section{References}

1. McGuigan A, Kelly P, Turkington RC, Jones C, Coleman HG and McCain RS: Pancreatic cancer: A review of clinical diagnosis, epidemiology, treatment and outcomes. World J Gastroenterol 24: 4846-4861, 2018.

2. Lohse I and Brothers SP: Pathogenesis and treatment of pancreatic cancer related pain. Anticancer Res 40: 1789-1796, 2020.

3. Kawakubo K, Tada M, Isayama H, Sasahira N, Nakai Y, Takahara N, Miyabayashi K, Yamamoto K, Mizubo S, Mohri D, et al: Risk for mortality from causes other than pancreatic cancer in patients with intraductal papillary mucinous neoplasm of the pancreas. Pancreas 42: 687-691, 2013.

4. Lee PJ, Podugu A, Wu D, Lee AC, Stevens T and Windsor JA: Preoperative biliary drainage in resectable pancreatic cancer: A systematic review and network meta-analysis. HPB (Oxford) 20: 477-486, 2018.

5. Bakens M, van Rijssen B, van Woerden V, Besselink M, Boerma D, Busch O, Dejong K, Gerhards M, van Hooft J, Keulemans Y, et al: Evaluation of preoperative biliary drainage in patients undergoing pancreatoduodenectomy for suspected pancreatic or periampullary cancer. J Pancreas 19: 24-28, 2018.

6. Brasoveanu V, Anghel C, Barbu I, Pautov M, Ionescu M, Motthor M, Balescu I, Dima S and Bacalbasa N: Pancreatoduodenectomy en bloc with portal and superior mesenteric artery resection-a case report and literature review. Anticancer Res 35: 1613-1618, 2015.

7. Bacalbasa N, Balescu I, Tanase A, Pautov M, Brezean I, Vilcu M and Brasoveanu V: Spleno-pancreatectomy En Bloc with parcelar gastrectomy for splenic artery aneurysm-a case report and literature review. In Vivo 32: 915-919, 2018.

8. Brasoveanu V, Dumitrascu T, Bacalbasa N and Zamfir R: Splenic artery used for replaced common hepatic artery reconstruction during pancreatoduodenectomy-a case report. Chirurgia (Bucur) 104: 499-504, 2009.

9. Bacalbasa N, Balescu I, Tanase A, Brezean I, Vilcu M and Brasoveanu V: Successful resection of a non-functional paraganglioma with celiac trunk invasion followed by common hepatic artery reimplantation-a case report and literature review. In Vivo 32: 911-914, 2018

10. Kawarabayashi N, Seki S, Hatsuse K, Kinoshita M, Takigawa T, Tsujimoto H, Kawabata T, Nakashima H, Shono S and Mochizuki H: Immunosuppression in the livers of mice with obstructive jaundice participates in their susceptibility to bacterial infection and tumor metastasis. Shock 33: 500-506, 2010.

11. Moole H, Bechtold M and Puli SR: Efficacy of preoperative biliary drainage in malignant obstructive jaundice: A meta-analysis and systematic review. World J Surg Oncol 14: $182,2016$.

12. Sewnath ME, Karsten TM, Prins MH, Rauws EJ, Obertop H and Gouma DJ: A meta-analysis on the efficacy of preoperative biliary drainage for tumors causing obstructive jaundice. Ann Surg 236: 17-27, 2002.

13. Ellison EC, van Aman ME and Carey LC: Preoperative transhepatic biliary decompression in pancreatic and periampullary cancer. World J Surg 8: 862-871, 1984.

14. Smith RA, Dajani K, Dodd S, Whelan P, Raraty M, Sutton R, Campbell F, Neoptolemos JP and Ghaneh P: Preoperative resolution of jaundice following biliary stenting predicts more favourable early survival in resected pancreatic ductal adenocarcinoma. Ann Surg Oncol 15: 3138-3146, 2008.

15. Peskova $\mathrm{M}$ and Gurlich R: Preoperative biliary drainage before pancreatoduodenectomy in patients with obstructive jaundice. Eur Surg 37: 331-335, 2005.

16. Garcia-Plata E, Seco JL, de la Plaza M, Vidal O, Alvarez MA, Botín IL and Santamaría JL: Influence of preoperative biliary drainage on postoperative morbidity and mortality after pancreatoduodenectomy. Cir Esp 77: 203-207, 2005 (In Spanish). 